\title{
Virtual Scenes Construction Promotes Traditional Chinese Art Preservation
}

\author{
Hui Liang 1[0000-1111-2222-3333], Fanyu Bao 2[1111-2222-3333-4444], Yusheng Sun ${ }^{3}$, \\ Chao $\mathrm{Ge}^{4}$, Jian Chang 5 \\ ${ }^{1234}$ Zhengzhou University of Light Industry, Zhengzhou, China \\ ${ }^{5}$ Bournemouth University, Poole, UK \\ hliangezzuli.edu.cn
}

\begin{abstract}
Chinese traditional opera is a valuable and fascinating heritage assert in the world as one of the most representative folk art in Chinese history. Its characteristic of 'suppositionality' in stage scenery provides a possibility of preservation of cultural heritage by digitization means, e.g., 3D Animation and Virtual Reality-based art show. In this novel digitization art form, the construction of virtual scenes is an important pillar--variety of created models should be accommodated to provide a vivid performance stage, including stage props and background. However, the generation of scenes based on traditional manual 3D virtual props modelling method is a tedious and strenuous task. In this paper, a novel shadow puppetry virtual stage scenes construction approach based on semantic and prior probability is proposed for the generation of compositional virtual scenes. First, primitive models based on semantics text segmentation and retrieval is provided for scene composition; and then, scene placement algorithm based on prior probability is conducive to assign these 3D models within virtual scene. This method is tested by generating the virtual performance stage for our shadow puppetry prototype system, within which various traditional art-specific 3D models are assembled. Its ease of use can assist artists to create visually plausible virtual stage without professional scene modelling skill. The user study indicates our approach's effectiveness and its efficiency.
\end{abstract}

Keywords: Virtual Scenes Construction, Traditional Chinese Art, Semantic.

\section{Introduction}

With a long history, the Chinese culture has always been a shining pearl in the history of mankind. As one of the unique traditional entertainment items in China, Chinese opera is an important part of traditional Chinese culture and known as the treasure of Chinese culture [1]. Shadow puppetry [2], figure silhouette made of animal skin or cardboard to tell folk stories, carries and contains the Chinese culture inherited from our ancestors. However, the form of shadow puppetry is more and more out of step with the rapid development of the times [3,4]. The inheritance of cultural heritage faces great challenges. New possibilities of heritage protection are provided for 
cultural digitization. Firstly, the digitized cultural heritage can be watched by users for a long time without interruption. Secondly, the loss, destruction and theft of historical relics can be prevented, so as to achieve the universality and permanent accessibility of cultural heritage [5]. Hence, we can realize the protection of traditional art through digital forms of expression [6].

The construction of virtual environment uses computer technology, multimedia technology, image processing technology and other technologies [7, 8]. 3D shadow puppetry stage Scene Generation is the core of the puppetry show, which will produce a better cultural heritage preservation effort by providing a vivid virtual scene art effect. With the introduction of digital technology, the construction of virtual scene is faced with the problems of complex technology, long production cycle and so on [9]. Our goal is to design scene placement algorithm based on semantic analysis and prior probability, improved the efficiency of scene automation construction.

The rest of this paper is structured as follow. Section 2 presents a background in compositional virtual scenes generation and some related works. Section 3 focuses on the design of scene primitive model generation and scene placement algorithm. Section 4 mainly discusses implementation of prototype system. Section 5 invited 30 college students of opera major volunteers to evaluate this system. Finally, Section 6 presents our main conclusions and some ideas for future work.

\section{$2 \quad$ Related Work}

The UK Culture Secretary, David Lammy, announced in Bristol the European Collaborative Action Plan on Digitisation of Cultural and Scientific Content to ensure better access to Europe's cultural heritage through the Internet [10]. The Central Opera House and The Beijing Institute of Technology set up the "Digital Stage Joint Laboratory". They use virtual reality to set the opera stage [11]. George Tsypin, as a master of international stage art design, has integrated 3D into the stage. An 8.2 meter $(27 \mathrm{ft})$ high and 27.4 meter $(90 \mathrm{ft})$ long semicircular ultrathin LED screen wall is used on the stage to further enhance the sense of reality and movement [12]. In the cultural heritage domain, one of the applications of virtual reality (VR) is the virtual reconstruction of the Forum of Augustus [13]. An interesting application is Arkaevision Arkeo. It is aimed at finding the temple of Hera in Paestum Posidona. During the game, users come to understand the culture of the Magnogreek city in the 5th Century BC [14].

In image reconstruction, Remondino et al described a method that uses a multistage image modeling approach for digital heritage preservation [15]. Song et al. [16] is the author of Semantic Scene Completion from a Single Depth Image. This method of automatic scene construction is commonly used in current animation scene generation.

Photogrammetry is used to generate scenario. When generate 3D reconstructed images, techniques such as SfM (Structure from Motion) and CRP (Close Range Photogrammetry) could be used to enhance the quality [17]. Balletti developed a system based on SfM and re-created a 3D model with digital camera [18]. 
Automatically generate scenarios using natural language processing. NLP (Natural Language Processing) within the AI programs dates back to the end of 80 s.The SHRDLU system used natural language to move various objects around in a closed virtual world [19]. An NLP-based 3D scene generation system for children with autism or mental retardation [20] offers great opportunities for disabled children to communicate with others.

To the best of our knowledge, one of the most innovative ways for 3D animation scene generation is the natural language processing[21]. In 2005, the 3DSV (3D Story Visualiser) system [22] was proposed by Zeng et al. They implemented an interactive 3D animation interface that builds a scene from a story text with simple constraints. Spika et al. [23] describe AVDT (Automatic Visualization of Descriptive Texts) for advanced scene production. Chang et al (2014) [24] is the developer of a text-to-scene system, they focused on learning dimension special by lots of indoor scenes. In their system, users can adjust models to match the text. Other than these, the WordsEye Text-to-Scene System has been developed about twenty years.

WordsEye [25] is a system which can automatically convert a natural language text into 3D scene representing. After two decades of development, the system has become very mature. A survey of the system is given in Ulinski and Coyne [26], shows that WordsEye has good performance. S2S is like WordsEye in countenancing entirely natural linguistic expressions as input. Nonetheless, S2S compared to WordsEye, it generates fairly less complicated scenarios [27].

In 2014, Stanford University proposed a scene generation system that can infer the implicit relationship in the text, and proposes to use conditional probability to obtain the relatively common model placement patterns [28]. For example, a static scene is generated with the statement "There is a room with a poster bed and a poster." (Fig.1).

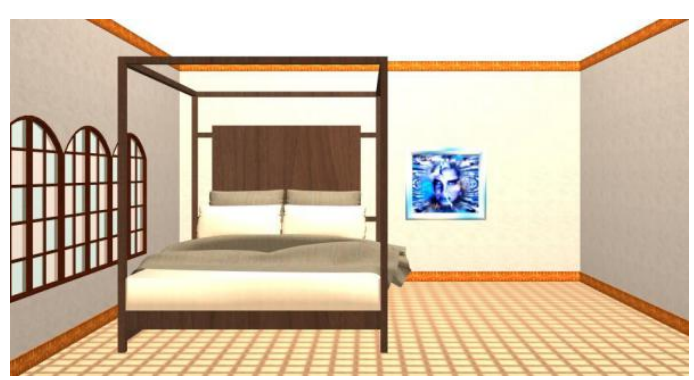

Fig. 1. Generated scene for "There is a room with a poster bed and a poster."

With the emergence of new technologies such as neural networks and machinelearning, natural language processing has enjoyed a continuous development. Natural language processing is a fusion of linguistics, computer science and artificial intelligence. It assists computers as close to human language, as possible to understand tasks. However, natural language processing has been widely used in text processing, it is still difficult to analyze specific text. The ignorance of internal semantic information would generate the semantic gap between input text and generated scene. In order to make generated scene suitable for the text which we input, we chose semantic analysis to process the text. 
This paper we use the semantic analysis for model generation and prior probability-based algorithm for scene generation. Our method enables to generate scene easily and also advance in bridging the aforementioned semantic gap, and provides a new method for cultural protection.

\section{$3 \quad$ Method}

\subsection{Scene primitive model generation based on semantics}

Scene primitive model generation based on semantics mainly consists of semantics text segmentation, model library, model generation and placement. Word segmentation algorithm is used for the semantic understanding and the acquisition of key words. Key words are imported into the model library to search for and automatically generate the desired model. The scene construction is completed through our proposed automatic placement algorithm of priori probability model suitable for traditional Chinese dramatic scenes.

The accuracy of text semantic understanding is the key to the success of the generation. The key words obtained from text segmentation directly determine the accuracy and correctness of the generation model. Due to the exquisite and sophisticated design of objects in the scene, the overall modeling effect is general, and the details cannot be highlighted. We use the semantic decomposition-combination method to build automatically. Relative to the use of spaces for word separation in English text, Chinese text will produce semantic noise when semantically segmented, which can easily produce multiple segmented results and cause ambiguity. Therefore, we use statistical word segmentation to build a Bi-Gram statistical model to count the frequency of text occurrence, and then use Hidden Markov Model (HMM) to extract key words correctly by learning and counting the occurrence of the same vocabulary in different texts several times.

(1) Text semantic statistics based on Bi-Gram

Assuming that $J$ represents a piece of text, the length is $\mathrm{n}$, and it is composed of $\left(M_{l}\right.$, $M_{2} \ldots M_{n}$ ) word sequence, then the probability of representing $J$ is:

$$
P(J)=P\left(M_{1}, M_{2} \ldots M_{n}\right)=P\left(M_{1}\right) * P\left(M_{2} \mid M_{1}\right) * \ldots * P\left(M_{n} \mid M_{1}, M_{2} \ldots M_{n-1}\right)
$$

Binary means that the word $M_{n}$ appearing in the nth is related to historical word $M_{n-1}$ Bi-Gram Mode:

$$
P\left(M_{1}, M_{2} \ldots M_{i}\right)=\prod_{n}^{i} P\left(M_{n} \mid M_{n-1}\right)
$$

(2) Word segmentation based on Hidden Markov Model (HMM) The HMM is a quintuple containing:

- Set of state values $Q=\left\{q_{1}, q_{2}, \ldots, q_{N}\right\}, N$ : possible number of states.

- Set of observations $V=\left\{v_{l}, v 2, \ldots, v_{M}\right\}, M$ : possible number of observations.

- Transition probability matrix $A=\left[a_{i j}\right] M^{*} N, a_{i j}$ : probability of transition from state $i$ to state $j$. 
- Observation probability matrix $B=\left[b_{j}(k)\right] M^{*} N, b_{j}(k)$ : Probability of generating observation vk under the condition of state value $j$.

- Initial state distribution $\pi$.

We use HMM for word segmentation. First, set the state value set $Q$ to $\{B, E, M, S\}$ to indicate the beginning, end, middle of a word, and the character-independent word (single). Then, for the string $C=\left\{c_{1} \ldots c_{n}\right\}$ to solve the maximum conditional probability: $\max P\left(t_{1} \ldots t_{n} \mid c_{1} \ldots c_{n}\right) . t 1$ denotes the state corresponding to the character c1. Eq (3) can get through Bayesian processing:

$$
P\left(t_{1} \ldots t_{n} \mid c_{1} \ldots c_{n}\right)=P\left(c_{1} \ldots c_{n} \mid t_{1} \ldots t_{n}\right) P\left(t_{1} \ldots t_{n}\right)
$$

Make a finite historic assumption: assume that the ci of the received signal at moment $i$ is determined only by the transmitted signal $t_{i} \mathrm{Eq}(4)$ :

$$
P\left(c_{1} \ldots c_{n} \mid t_{1} \ldots t_{n}\right)=P\left(c_{1} \mid t_{l}\right) P\left(t_{l}\right) \ldots P\left(c_{n} \mid t_{n}\right) P\left(t_{n} \mid t_{n-1}\right)
$$

Finally, Viterbi algorithm is used to find the final optimal word segmentation sequence.

$$
\delta_{t+1}(i)=\max \left[\delta_{t}(j) a_{i j}\right] b_{i}\left(c_{t}+1\right)
$$

$\delta_{t}(j)$ : Maximum probability of state $i$ at time $t$.

In the scene of 'The Emperor and the Assassin', Mrs. Xu's dagger is an important primitive model. The short sword, which is divided into three parts: the body of the dagger is a short silver edge, the handle is brown, and the tip of the dagger is golden yellow. So, the Chinese text to be analyzed is '我需要一柄银色的剑身 (I need a sword with silver sword body), 棕色的剑柄 (brown sword handle), 金黄色的剑梢的 剑 (golden sword tip)'. The '银色的剑 (Silver sword)', '棕色的剑 (Brown sword)', '金黄色的剑 (Golden sword)' are text ambiguities. By building a Bi-Gram Model, we get P (剑身 (Sword body) | 银色 (Silver)), P (剑柄 (Sword handle) | 棕色 (Brown)), P (剑梢 (Sword tip) | 金黄色 (Golden)), and P(剑(Sword)| 银色 (Silver)), $\mathrm{P}$ (剑 (Sword)| 棕色 (Brown)), P(剑 (Sword)|金色 (Golden)). Then use the Chinese word breaker corpus for training HMM, through supervised learning, get silver-white modified sword body, not modified sword. Finally, use the trained HMM treatment to get '我 (I) / 需要 (need) / 一柄 (a) / 银色的 (Silver) / 剑身 (Sword body) /, / 棕色 的 (Brown)/剑柄 (Sword handle)/, / 金黄色的 (Golden)/剑梢 (Sword tip)/的 (a) / 剑 (Sword) $/$ 。'.

The key words are extracted from the semantic understanding, then the threedimensional model library of the stored entity is searched by the keywords. We store the basic information of the primitive model in a JSON file format for easy export of accurate primitive models. The fields of the primitive model information table include: Name, Towards, Color, Format. The primitive model information table is shown in Table 1. 
Table 1. Attribute information of primitive model

\begin{tabular}{ll}
\hline & \multicolumn{1}{c}{ Attribute information } \\
\hline Name & 剑身 \\
Towards & towards.x, towards.y, towards.z \\
Color & 银色 \\
Size & Length, width, height \\
Format & Obj, 3ds, stl, fbx, mb, stp, igs, max, 3ds, ma, vtk \\
Position & Position.x, Position.y, Position.z \\
\hline
\end{tabular}

Primitive model generation searches the model library for keyword matches based on entity names extracted from the previous section. The primitive model is automatically generation as shown in Fig. 2.

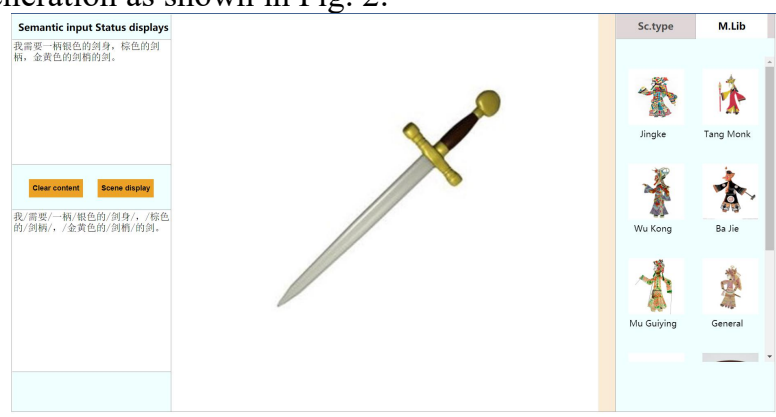

Fig. 2. Primitive model is automatically generation

\subsection{Scene placement algorithm based on priori probability}

In view of the particularity of Chinese traditional opera scene, there are a lot of common sense logical spatial relations in the scene that match with the prior probability algorithm model. Based on the statistics of the basic placement and spatial relationship of the primitive model in the scene, the data probability model is designed. On this basis, the model placement algorithm based on prior probability is proposed innovatively. The spatial model is transferred to the spatial model as a prior spatial model through JSON. The model space area table is shown in Table 2.

Table 2. Three-dimensional spatial area table.

\begin{tabular}{ll}
\hline & \multicolumn{1}{c}{ Spatial region } \\
\hline Center & Center.x, Center.y, Center.z \\
RelPos & relPos.x, relPos.y, relPos.z \\
Semantics & 上 \\
Probability & 0.68 \\
\hline
\end{tabular}

This paper analyzes the text semantics of spatial relations of primitive models and extracts the spatial relations of matched indoor scenes [29]. Spatial relations are the orientation relations between models. Because the spatial positions generally need 
three points to be determined, a triple set between primitive models is constructed. For example: Mrs. Xu's dagger is on the top of the map of the state of Yan, and the table for offering is under the map of the state of Yan. The table is located in the north of the hall, and to the west is a carved screen. It forms a triple set

\{

(map of Yan state, upper part, Mrs. Xu's dagger),

(Altar, map of Yan state),

(Altar, north side, Audience Hall),

(Altar, west side, screen)

\}.

First, the coordinate system needs to be determined, with the spatial Cartesian coordinate system generated by the spatial relationship of the scene as the world coordinate system, the absolute orientation. Converts the obtained spatial location to a JSON file in the same format as Table 1 and matches the strings; matches the successful output file and translates the spatial shift to the specified location by converting the JSON file to model information. Failed matching queries several locations of related models in Table 1, compares probability attributes, and outputs the maximum probability [30]. The resulting scene description file JSON format is converted:

\{

File information: \{version number, type $\}$,

Scene type: $\{$ The palace of the Qin Dynasty, Audience Hall\}

Scene: \{

Name: 'Altar',

Scene: 'Audience Hall', Position: $\{x, y, z\}$

Size: $\{\mathrm{x}, \mathrm{y}, \mathrm{z}\}$,

\}

\}

Finally, the generated scene description file is submitted to Three.js to analyze. Three.js to analyze the JSON file and querying the model information contained in the file, in order to improve the efficiency of model loading, the system will first determine whether the model is contained in the local cache. If it exists, it will be called directly. If not, it needs to be obtained from the server. Then the size and coordinate position information in JSON file is combined with the model to render and display in the page. Fig.3 shows the result after parsing the statement '卧室的墙 边有一张床 (There is a bed by the wall of the bedroom)'. As can be seen from the figure, for the common objects, the system can achieve the expected results.

\{“FileInfo": \{“version": 1.0, "type": "JSON"\},

"GroundType": "flat",

“Object”: \{“Name”: “床”,

"Scene": “客厅”,

"Position": \{“x": 1.33, “y": 2.66, "z": 3.67$\}$,

"Scale": \{“x": 1, "y": 1 , "z": 1$\}$,

"Rotation": \{“rot": 0, "yaw": 0, "pitch": 0$\}\}\}$ 


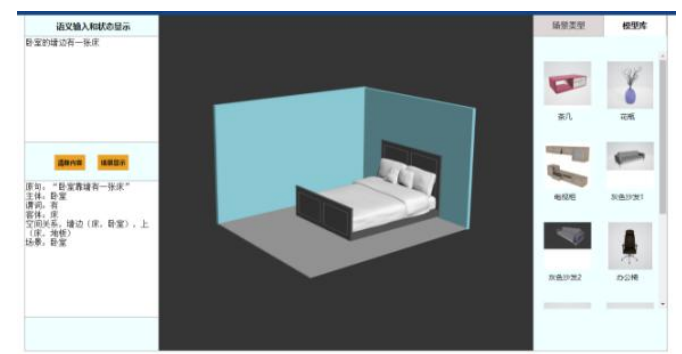

Fig. 3. Generated scene for '卧室的墙边有一张床 (There is a bed by the wall of the bedroom)'

\section{$4 \quad$ Implementation}

Chinese traditional opera is a stage performance art that combines literary, music, martial arts, performance, acrobatics and other elements. Performers, on a specific stage, talk about China's long history through the combination of singing and action. Stage scene layout is very important to the success of a dramatic performance.

\subsection{Realization of Scene primitive model}

Use the word segmentation algorithm to obtain keywords which the information about the placement of objects in the scene, such as subject, object, predicate, spatial orientation relationship, scale ratio in the text, and find similar characters in the model library. We choose The Emperor and The Assassin as an example of placing objects in the scene. In the process of scene modeling, the spatial relationship between different objects is the focus of our placement. We have made a statistic on the placement relation between common objects, which is convenient for reference later, so as to improve the accuracy of object placement, enhance the practicability of the system, and the interactivity of users. For example, the input text '我需要戴着红色的 戏帽翎 (I need to wear the plume of the red opera hat's plumes), 黄色的戏帽顶 (the top of the yellow opera hat's top), 红色的戏帽檐的帽子头部 (the head of the red opera hat with the red opera hat's brim), 下面身体是红色黄色为主的戏服的人 (and the body below is the person in the costume with the main color of red and yellow)。'. The text analysis and generation objects are shown in the figure below. $\mathrm{P}$ (戏帽翎 (Opera hat's plumes) | 红色 (Red)), P (戏帽顶 (Opera hat's top)|黄色 (Yellow)), P (戏帽檐 (Opera hat's brim) | 红色 (Red)) 和P (戏帽 (Opera hat)|红色 (Red)), P (戏帽 (Opera hat) | 黄色 (Yellow)), P (戏帽 (Opera hat) | 红色 (Red)) are gained through Bi-Gram text statistics. Through HMM word segmentation processing, I finally got “我 (I)/需要 (need to)/戴 (wear)/着(the)/红色的(red)/戏帽 翎 (opera hat's plumes) /, /黄色的 (yellow) /戏帽顶(opera hat's top) /, /红色的(red) /戏帽檐 (opera hat's brim) /的 (of)/帽子 (hat)/头部(heat)/, /下面 (below)/身体 (the body) / 是(is)/红色(red)/黄色 (yellow)/为 (with)/主 (main)/的 (of) / 戏服 (costume) /的 (of) / 人(person)/。'. Import keywords such as '戏帽翎 (Opera hat's plumes), 黄 色的 (Yellow), 戏帽顶 (Opera hat's top), 红色的 (Red), 戏帽檐 (Opera hat's 
brim), 帽子 (Hat)' and other keywords into the model library, and finally obtain the required model (Fig.4).

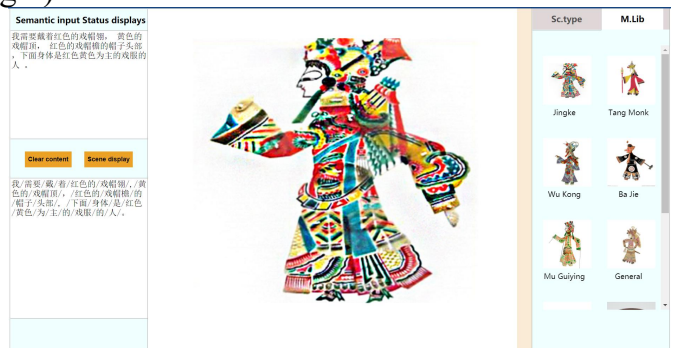

Fig. 4. Text analysis and model generation

\subsection{Scene placement}

Scene placement is the top priority in scene construction, which directly reflects the effect presented by the scene. The corresponding model is found in the model library according to the objects in the text, and the coordinate information of each model is calculated by using prior probability according to the spatial relationship between objects, and then the coordinate information is combined with the model attribute information extracted previously. With the shadow puppetry of China's intangible cultural heritage as the background, we get an unordered set of Triple through prior probability:

(PanLong column, East, King Qin)

(King Qin, East, Jing Ke)

(Screen, North, Jing Ke)

Then convert it into a JSON file and import it into the database for string matching, and finally export the required placement position. 'The Emperor and the Assassin' is completed as shown in Fig. 5.

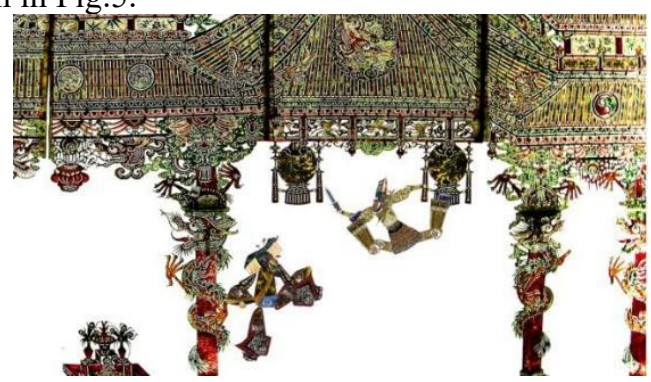

Fig. 5. 'The Emperor and the Assassin' scene generation

\section{$5 \quad$ Experiment \& Discussion}

We use Leap Motion to track and control the gesture of hand movement, and gesture replaces the bamboo stick in Chinese traditional opera to directly control the movement of shadow puppetry [31-33]. The realization of human-computer interaction can not only restore image and speech, but also realize multi-angle and multi-directional viewing. Digital shadow puppetry provides us with new ideas and 
methods to solve the problem of high production difficulty and long-term preservation.

A set of questions is designed to assess student's understanding of traditional opera, their acceptance of virtual reality, the effect of virtual scene construction, the operation, etc.. 30 college students of opera major volunteers were invited to experience our prototype for evaluation. After the experiment, questionnaire surveys were sent to all the participants to give feedback on the practicability of the digital stage. The findings in Fig.6 show that some students may be new to virtual reality games and are not very proficient in virtual reality games. Compared with traditional opera performance, they are more like the novel and immersive VR-based performance environment. Most of the students think that the digital stage enhances the stage performance and is helpful to the study of opera culture.

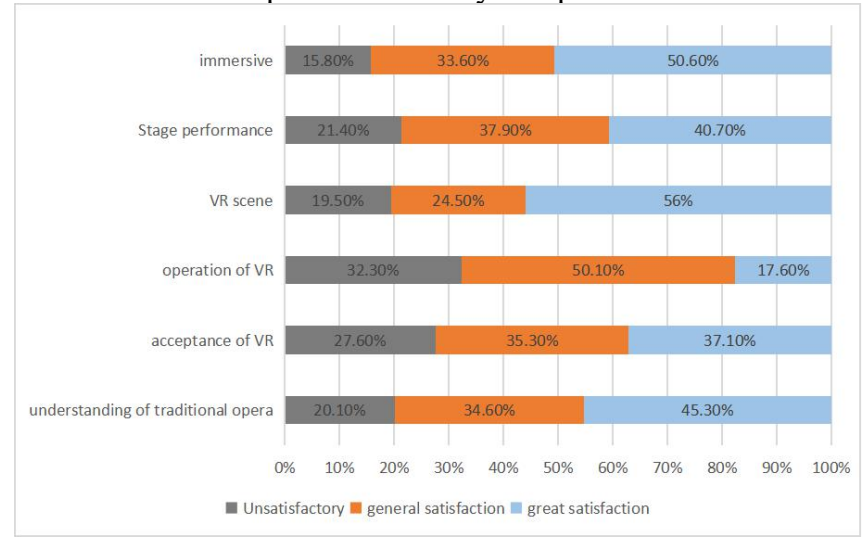

Fig. 6. Distribution of survey results

Combining all the feedback from the questionnaire leave message, users' suggestions can be summarized into three positive points, which are

- The combination of VR and opera is more attractive to students;

- It provides a new possibility for the inheritance of Chinese traditional opera culture;

- The automatic construction effect of virtual scene is remarkable and provides a good stage performance.

\section{Conclusion}

We use semantic-based scene original model generation and our prior probabilitybased scene placement algorithm to complete the scene generation. According to the generated results, the output scene and the demo text are highly similar. Experiments have proved that the scene placement algorithm based on prior probability can save time and reduce the difficulty of modeling. However, due to the limited number of models in the model library, it is difficult for some objects to find a fully corresponding model, and only the closest feature can be selected object. In the future research process, we plan to establish a community where everyone can upload and 
download models from the website to solve the problem of insufficient models in the model library. We will continue to combine more fields with advanced technology to enrich digital content.

\section{References}

1. Yan, S., Qiang, W.: The Experience Design of Shadow Puppet Culture Based on Tangible Interaction. In: Proceedings of 2016 Nicograph International, pp. 64-67. (2016).

2. Dostal, C., Yamafune, K.: Photogrammetric texture mapping: A method for increasing the Fidelity of 3D models of cultural heritage materials. Archaeol. Sci. Reports, vol. 18, pp. 430-436. (2018).

3. Zhang, Y., Fangbemi, A.: "Third-Person" Augmented Reality-Based Interactive Chinese Drama, In: Proceedings of 2015 International Conference on Culture and Computing (Culture Computing), pp. 41-46. (2015).

4. York Q.Y., Shen J.H., Yang Y.: How 'dama' becomes drama-assessing the ideological forces underlying open air group dances by Chinese senior females. Lsure Studies, pp. 110. (2019).

5. Porte, B.D.L., Higgs, R.: Challenges in digitisation of cultural heritage material in the Western Cape, South Africa. SA Journal of Information Management, vol.21 no.1, pp. 111. (2019).

6. Haval, N.: Three-dimensional documentation of complex heritage structures. IEEE MultiMedia, vol. 7, no. 2, pp. 52-55. (2000).

7. Wang, Y., Hu, X.: Wuju Opera Cultural Creative Products and Research on Visual Image Under VR Technology. IEEE Access, vol. 8, pp. 161862-161871. (2020).

8. Deng, F., Gan, B.: Research on the Application of 3D Virtual Simulation Technology in Ancient Village Restoration. In: Proceedings of 2019 4th International Conference on Mechanical, Control and Computer Engineering (ICMCCE), pp. 1050-10503. (2019).

9. Bastug, E., Bennis, M., et al.: Toward Interconnected Virtual Reality. Opportunities, Challenges, and Enablers. IEEE Communications Magazine, vol. 55, no. 6, pp. 110-117. (2017).

10. Francesco, G.D.: Towards an agreed European common platform for digitisation of cultural and cientific heritage: The ministerial network for valorising activities in digitisation (MINERVA). Review of the National Center for Digitization, pp. 2-11. (2005).

11. Zhang, Y.X., Zhu, Z.Q.: On Stage Interactive Spatial AR for Drama Performance. In: Proceedings of 2016 IEEE International Symposium on Mixed and Augmented Reality (ISMAR-Adjunct), pp. 280-283. (2016)

12. Zhang, F., Ding, G., et al.: Research of simulation of creative stage scene based on the 3DGans technology. Information Hiding and Multimedia Signal Processing, vol. 9, no. 6, pp.1430-1443. (2018).

13. Ferdani, D., Fanini, B., et al.: 3D reconstruction and validation of historical background for immersive VR applications and games: The case study of the Forum of Augustus in Rome. Cultural Heritage, vol. 43, pp. 129-143. (2020).

14. Bozzelli, G., Raia, A., et al.: An integrated vr/ar framework for user-centric interactive experience of cultural heritage: the arkaevision project, Digital Applications in Archaeology and Cultural Heritage, vol. 15. (2019).

15. Remondino, F., El-Hakim, S.F., et al.: Turning images into 3D model. IEEE Signal Processing Magazine, vol. 25, no. 4, pp. 55-65. (2008). 
16. Song, S., Yu, F., Zeng, A., et al.: Semantic Scene Completion from a Single Depth Image. In: Proceedings of 2017 IEEE Conference on Computer Vision and Pattern Recognition (CVPR), pp. 190-198. (2017).

17. Fonstad, M.A., Dietrich, J.T., et al.: Topographic structure from motion: a new development in photogrammetric measurement. Earth Surface Processes \& Landforms, vol. 38, no. 4, pp. 421-430. (2013).

18. Ba Lletti, C., Guerra, F.: The survey of cultural heritage: a long story. Rendiconti Lincei, vol. 26, pp. 115-125. (2015)

19. Winograd, T.: Understanding Natural Language. Massachusetts Insti-tute of Technology, vol. 3, no. 1, pp. 115-125. (1972).

20. Kiliçaslan, Y., et al.: An NLP-Based 3D Scene Generation System for Children with Autism or Mental Retardation. In: Proceedings of Artificial Intelligence and Soft Computing - ICAISC 2008, pp. 929-938. (2008).

21. Coyne, B., Sproat, R.: WordsEye: An automatic text-to-scene conversion system. In: Proceedings of 28th Annual Conference on Computer Graphics and Interactive Techniques (SIGGRAPH 2001), pp. 487 - 496. (2001).

22. Zeng, X., Mehdi, Q., Gough, N.: From Visual Semantic Parameterization to Graphic Visualization. In: Proceedings of Ninth International Conference on Information Visualisation (IV'05), pp.488-493. (2005)

23. Liu, D., Bober, M., Kittler, J.: Visual Semantic Information Pursuit: A Survey. IEEE Transactions on Pattern Analysis and Machine Intelligence, vol. 43, no. 4, pp. 1404-1422, (2021).

24. Chang, A.X., Savva, M., Manning, C.D.: Semantic parsing for text to 3D scene generation. In: Proceedings of Asso-ciation for Computational Linguistics (ACL) Workshop on Semantic Parsing, pp. 17-21. (2014).

25. Spika, C., Schwarz, K., et al.: AVDT - Automatic Visualization of Descriptive Texts. In: Proceedings of the Vision, Modeling, and Visualization Workshop 2011, pp. 129- 136. (2011).

26. Ulinski, M., Coyne, B., Hirschberg, J.: Evaluating the WordsEye Text-to-SceneSystem: Imaginative and Realistic Sentences. In: Proceedings of the Eleventh International Conference on Language Resources and Evaluation (LREC 2018), pp. 1493-1499. (2018).

27. Milliet, Q.: Virtual Reality and 3D Animation in Forensic Visualization. Forensic Sciences, vol. 55, no. 5, pp. 1227-1231. (2011)

28. Chang, A.X., Savva, M., Manning, C.D.: Learning Spatial Knowledge for Text to 3D Scene Generation. In: Proceedings of the 2014 Conference on Empirical Methods in Natural Language Processing (EMNLP), pp. 2028-2038. (2014)

29. Lin, Y., François, C.: Chinese Opera and the International Market. Arts Management, vol. 20, no. 3, pp. 75-82. (2018).

30. Brunetaud, X., Janvier, R., et al.: The Valmod Project: Historical and Realistic 3D Models for the Touristic Development of the Château de Chambord. In: Proceedings of EuroMediterranean Conference, vol 10059, pp. 53-60. (2016).

31. Liang, H., Chang, J., et al.: Hand Gesture Based Interactive Puppetry System to Assist Storytelling for Children, The Visual Computer, vol 33, no. 3, pp. 31-15. (2017)

32. Liang, H., Zhang, Q., et al.: Surface modelling of Jun ware based on ordinary differential equations. Traitement du Signal, vol. 36, no. 1, pp. 53-58. (2019)

33. Wu, F.L., Liang, H., et al.: Semantic Framework Promotes Interactive Puppetry Animation Production to Assist Storytelling Training. Information Science and Engineering, vol. 36 no. 6, pp. 1179-1189. (2020) 